\title{
PROBLEMAS DE LA COMPRENSIÓN LECTORA EN LA EDUCACIÓN RURAL
}

\author{
Srta. Lourdes Romero Jiménez
}

\begin{abstract}
RESUMEN
La diversidad del Perú es un reto, más aún, desde un punto de vista educativo porque las diferencias afectan el aprendizaje. Por esto, los niños de zonas rurales resultan con menores posibilidades de una educación consistente, dadas las limitaciones propias de su entorno y las condiciones de vida. Toca realizar proyectos

educativos con una auténtica incorporación de variables capaces de revertir esa situación.
\end{abstract}

\section{ABSTRACT}

Cultural diversity of Peru is a challenge, even more since an educational point of view, because cultural differences affect learning. Far such fact, children from rural areas have fewer possibilities to have access to an adequate education, considering the limitations of their environment and life conditions. It is time to develop educational project with an authentic incorporation of variables able to change this situation.

\section{PALABRAS CLAVE}

Comprensión, lectura, escuela rural, diseño curricular, lengua.

\section{KEYWORDS}

Comprehension, reading, rural school, curriculardesign, language.

\begin{abstract}
En el 2010 se realizó la Prueba del Ministerio de Educación _ en todo el país, el cual evaluó la comprensión lectora en los alumnos del segundo grado de primaria. Según los resultados de la Evaluación Censal de Escolares del 2010, indica que más del 28\% alcanzó un nivel esperado y hubo un aumento de 5,6\% en comparación con la prueba del 2009, pero se resaltó que esta mejora es significativa solo en lima, Callao, Arequipa, lea y Lambayeque (Luis Zuta Dávila lzuta, 2011).
\end{abstract}

Es decir, las demás provincias no están dentro de la mejora. Los alumnos de las zonas rurales no presentan una significativa mejoría y las pruebas realizadas en años anteriores han dado como resultado cifras alarmantes en Comprensión Lectora.

¿Por qué los niños de segundo grado de primaria de las zonas rurales no obtuvieron un aumento significativo en el puntaje de la prueba de comprensión lectora del Ministerio de Educación del2010?

Se plantean diferentes hipótesis para esta pregunta, una de ellas es la falta de descentralización del sistema educativo peruano, ya que este sistema no es igualitario para todos los alumnos de diferentes zonas, teniendo como consecuencia que en las zonas rurales no exista una educación con calidad.

La falta de capacitación y acompañamiento a los profesores de las zonas rurales, ya que algunos de ellos no son de la propia comunidad, vienen de zonas urbanas y no se encuentran debidamente preparados para poder adaptar el Diseño Curricular Nacional a la realidad de los alumnos o no desarrollan las capacidades es"ablecidas y no suelen utilizar estrategias y métodos adecuados para el proceso de 
aprend izaje-enseñanza.

La desnutrición crónica que padecen los alumnos de zonas rurales. Como sabemos, la nutrición tiene una estrecha relación con el rendimiento académico de los alumnos, y esta población sufre de déficit de hierro y zinc, entonces lcómo esperamos que estos niños aprendan?, si cuando llegan a la escuela ya comienzan a sentir cansancio y no prestan la adecuada atención para poder realizar el proceso de la comprensión lectora.

La asistencia irregular de los alumnos a la escuela, es otra hipótesis planteada, ya que faltan en tiempo de cosecha, pues las familias tienen como actividad económica la agricultura y los niños tienen que trabajar para poder ayudar a sus familias económicamente. También, hay que tener en cuenta la asistencia irregular de los profesores de educación rural a la escuela.

\section{La falta de descentralización en el sistema educativo peruano}

Siempre se ha juzgado la centralización de nuestro sistema educativo, por ello, se realizó diferentes reformas y se aprobaron leyes que buscan la descentralización dela educación.

Se comenzó a reestructurar todo el sistema, buscando la independencia de cada región o provincia con respecto a la educación y puedan adaptar el Diseño Curricular Nacional (DCN) de acuerdo a su propia realidad educativa. También se habló de la Municipalización de la Educación, pero primero se dio Planes Pilotos de Municipalización que no tuvieron mucha relevancia.

SegúnIguiñiz(2008,p.42), paraladescentralización se ha transferido funciones y deberes escritos en reformasyleyes, peronosecumplencabalmente ono se ponen en práctica, lo cual nos quiere decir que la descentralización del sistema educativo no se ha dado por completo.

Hay querecalcar la desigualdad deeducación, ya que es un tema vinculado a la descentralización, como nos dice Muñoz (2008, p. 45), "no debemos olvidar que junto con la promoción de la descentralización porpartedelEstado, hayen el uncompromisomayor: el aseguramiento de la equidad, con el que se podrá garantizar el achicamiento de las brechas de desigualdad".

La desigualdad se refleja en la falta de una educación de calidad en las escuelas rurales, pues no cuentan con la infraestructura, los servicios y entornos adecuados en salubridad que tienen que brindarse satisfactoriamente para que los alumnos puedan tener un buen aprendizaje, como lo señala el Proyecto Educativo Nacional, ya que "la mayoría de las escuelas rurales no cuentan con una buena infraestructura sino que requieren reparaciones, y no cuentan con los tres servicios básicos: electricidad, agua y desagüe" (Benavides y otros, 2010, p. 95), creando así un malestar o incomodidad en los alumnos ylos maestros .

Las direcciones regionales sólo se encargan de establecer la descentralización de poderes o funciones y no de que todos reciban una educación por igual, ya que la prueba demuestra que hay una mejor calidad educativa en las escuelas urbanas que las rurales, ya que si hablaríamos de una igualdad, estas pruebas no tendrían cifras tan distanciadas en comparación con los de escuela urbana.

Lo que ocurre es que la gestión de la escuela rural tiene la perspectiva dirigida hacia afuera, primero quiere resolver las demandas formales del sistema de gestión local, regional y nacional o hacia el interés y las necesidades particulares de su personal; es decir, su atención no está hacia adentro, primero debe atender las necesidades de los niños y sus posibilidades deaprendizaje.

Por lo tanto, la descentralización no solo implica en delegar funciones, en crear programas o establecer leyes o reformas, sino que éstas sean concretizadas y puestas en práctica para que halle una verdadera descentralización educativa $y$, por ende, equidad en la educación.

\section{Capacitación y acompañamiento a los profesores}

Una escuela rural cuenta con profesores que son de zonas urbanas y que por primera vez van a enseñar en la escuela rural y algunos profesores que pertenecen a la misma población, que regresan después de haber culminado su estudio profesional. Según Carmen Montero (2001) se ha detectado que ambos profesores no han recibido la adecuada formación profesional para poder trabajar en escuelas rurales, ya que no cuentan con la calidad y cantidad suficiente de información o con las metodologías necesarias para poder realizar el proceso de enseñanza-aprendi zaje, sabiendo que muchos de ellos atienden aulas multigrado o donde el director también es el profesor del aula, lo que implica que tiene que ejercer las cinco funciones que la ley le da,

\section{1 un1Fé}


las siete funciones como profesor y garantizar las catorce que se asigna a la institución; hay que resaltar que los profesores tratan de poner todo su empeño para que los alumnos sean atendidos, pero hay momentos donde algunos alumnos de un determinado grado se queda con espacios vacíos o no se logra terminar el tema de enseñanza, dejando información incompleta.

Según la investigación de Carmen Montero (2001),se ha observado que algunas veces, los profesores brindan información equivocada sobre diferentes temas, suelen cometer errores en dar alguna explicación o en resolver operaciones aritméticas simples, como también los constantes errores ortográficos o problemas con la redacción de párrafos u oraciones sencillas escritas en castellano.

A la par, también se observa que los profesores de las escuelas rurales no preparan su clase con anticipación y no programan los temas a tratar en un tiempo determinado, lo que implica que no se prepara los materiales que van a utilizar o van a ayudar a que se pueda dar un aprendizaje significado, necesario en el nivel primaria; entonces, tal vez no se están brindando o aplicando ejercicios para una buena comprensión lectora, especialmente en primer o segundo grado, donde es necesario que se cree un hábito delectura en los niños.

Todo lo mencionado anteriormente, está causado por la falta de capacitación al profesor y la práctica de la política de acompañamiento a los profesores sobre estrategias y métodos viables para la enseñanza en las escuelas rurales o en las adaptaciones al DCN que se tienen que realizar, de acuerdo a la realidad de cada comunidad, las cuales no son realizadas por la falta de conocimiento o porque simplemente no creen que sean necesarios, ya que no hay un control constante hacía los profesores y algunos de ellos se quedan en lo rutinario o no ponen atención en las necesidades de los alumnos, haciendo que las clases sean aburridas pues son improvisadas y no cuentan con el material necesario. Por ello, se busca que se dé un asesoramiento metodológico, como a la vez, enriquecer, diversificar y actualizar el repertorio de recursos didácticos de los profesores (Consejo Nacional de Educación, 2008).

Hay que mencionar la falta de trabajo con el DCN por parte de los profesores, ya que algunas veces no se logra desarrollar las capacidades de las diferentes áreas establecidas, pues algunas de las capacidades, especialmente del área de Comunicación, se toman en cuenta en la prueba de comprensión lectora. Silos niños no desarrollan las capacidades planteadas entonces va a ser difícil que pueda responder satisfactoriamente a los indicadores de logro que están establecidos enla prueba.

los logros que se quieren obtener en el aprendizaje están postergados por las necesidades del docente, como: "asegurar su dominio de los conocimientos disciplinares y las habilidades básicas en diversas áreas, las que deben ser enseñadas a los estudiantes; desarrollar las competencias pedagógicas que le van a permitir conocer los saberes diferentes en un aula; estimular la reflexión crítica y la creatividad" (Consejo Nacional de Educación, 2008, p. 27), lo que va a ser necesario para que el alumno pueda rendir la prueba de comprensión lectora, ya que ésta también califica su actitud crítica y de reflexión en los diferentes tipos de textos presentados en la prueba.

\section{la desnutrición}

la desnutrición en nuestra población escolar rural, secuelas a corto plazo, se dan en la etapa escolar, especialmente en primaria, esto trae que las consecuencias se acumulen y aumenten las probabilidades de que el niño no aprenda y se da una desventaja educativa, ya que tiene una deficiencia de proteínas, micronutrientes, como elhierro yelzinc.

Según Ernesto Pollit (2005) la desnutrición en los niños trae efectos directos, ya que implica alteraciones cerebrales, que a la vez, producen cambios cognitivos, lo cual también afecta la motivación del niño haciendo que no aproveche las aprendizajes impartidos, pero hay que tener en cuenta, que varios de estos niños presentan situaciones graves, pues tienen una desnutrición temprana y continúa desnutrido durante la etapa escolar.

Por ello, ésta puede ser una causa razonable para el bajo rendimiento académico delos niños de escuelas rurales, ya que no va a poder realizar satisfactoriamente el proceso de cognición. Por ejemplo, una niña tiene que caminar aproximadamente dos kilómetros para llegar a una escuela (la más cercana a su casa), llega cansada a la escuela, el poco alimento que consumió ya se agotóy perdió energía, Icómo esperamos que la niña responda si ya perdió energías y tal vez hasta tenga hambre?

Para poder desarrollar una buena comprens1on lectora, necesitamos 4ue los niños tengan la atención y concentración adecuada, pues para ello necesitan 
las inasistencias es un problema en el cual se puede encontrar una solución favorable, tal vez adecuando el horario escolar a la realidad de la comunidad, es decir, para poder evitar que haya inasistencias por parte de los alumnos, y que los profesores se comprometan con su profesión, que puedan entender un día perdido de clases es un día perdido de aprendizaje del alumno, y que algunas veces prefieren recuperar el tema de enseñanza que practicar la comprensión lectora o poner en práctica el plan lector, establecido por el Ministerio de Educación .

Todo lo establecido anteriormente, trae como consecuencia que los alumnos de educación rural obtengan puntuaciones significativamente mínimas de mejoría, ya que el Estado tiene que poner más énfasis en la educación rural que se encontraba olvidada y recién se está trabajando para poder establecer una equidad educativa en todo el territorio peruano.

\section{REFERENCIAS}

Benavídes, Martín; Mena, Magríth y Ponce, Carmen (2010). Estado de la niñez indígena en d Perú. lima: Instituto Nacional deEstadística e Informática(INEI) y Fondo de las Naciones Unidas para la Infancia (UNICEF).

Consejo Nacional de Educación (2008). Programa de acompañamiento pedagógico para mejorar aprendizajes en las instituciones educativas de áreas rurales. lima: Proyecto AprenDes.

Dávila lzuta, luís Zuta (2011). Más estudiantes mejoraron en comprensión lectora. Diario Oficial El Peruano, № 23317. Disponible

e n : htt p://www.elperuano.com.pe/edícíon /notícia.aspx? key=vFNXY0xl/Mk Consultado el 27-5-11.
Dávíla, Réne (2009). iDesnutricióni Problemas de aprendizaje y confusión al leery escribir.JOURNALMEX Periodistas de México. Disponible en: htt p://journalmex.wordpress.com/2009/08/25/ ${ }^{\circ}$ loC2 \% A ld esn u trícíon \%C 2 \% Al - probl emas -de a prendíza je-y-confusíon-al-leer -y -escríbír / Consultado el 3-6-11.

Fundación Inka Kola e Instituto Apoyo (2005). La educación rural en el Perú: problemas, propuestas, experiencias, esperanzas. lima: Címagraf.

Iguíñíz Echeverria, Manuel (2008). Descentralización del sistema educativo. lima: Tarea.

Montero, Carmen (2001). La escuela rural: modalidades y prioridades de intervención. lima: Programa Especial de MejoramientodelaCalidaddelaEducaciónPeruana.

Srta. Lourdes Romero Jiménez

Estudiante del IX ciclo del Programa Académico de Educación Especial, especialidad Retardo Mental. 